\title{
Postoperative Pulmonary Function in Open versus Laparoscopic Cholecystectomy: A Meta-Analysis of the Tiffenau Index
}

\author{
Gianfranco Damiani $^{a} \quad$ Luigi Pinnarellib $^{\text {a }}$ Annalisa Sammarco ${ }^{a}$ \\ Lorenzo Sommella $^{b}$ Marsilio Francuccic ${ }^{c}$ Walter Ricciardi ${ }^{a}$ \\ ${ }^{a}$ Department of Public Health, Università Cattolica S. Cuore and ${ }^{b}$ Hospital Trust San Filippo Neri, Rome, and \\ ${ }^{\mathrm{C}}$ Hospital Trust Santa Maria, Terni, Italy
}

\author{
Key Words \\ Open cholecystectomy • Laparoscopic cholecystectomy • \\ Pulmonary function - Forced expiratory volume in \\ 1 second · Forced vital capacity - Meta-analysis • \\ Tiffenau Index
}

\begin{abstract}
Background: Available scientific literature about open cholecystectomy (OC) and laparoscopic cholecystectomy (LC) does not show univocal results in terms of postoperative pulmonary function. A meta-analysis was carried out to evaluate the postoperative pulmonary function after OC and LC focusing on the Tiffenau index. Methods: Electronic databases were consulted (Cochrane Library, Embase and Pubmed). Standardized mean difference (SMD) with 95\% Cl was calculated for the Tiffenau Index. The kappa test was performed to evaluate agreement between the reviewers regarding the quality of the selected studies. A sensitivity analysis was carried out to assess the robustness of our study. Heterogeneity among studies was tested by using a $\chi^{2}$ test at 0.05 significance level. A random effects model metaanalysis was performed. Results: Meta-analysis of the 13 articles included, resulted in a SMD of $53 \%(95 \% \mathrm{Cl} 0.04,1.02)$ for the Tiffenau Index in favor of laparoscopic cholecystec-
\end{abstract}

\section{KARGER}

Fax +41613061234

E-Mail karger@karger.ch

www.karger.com (c) 2008 S. Karger AG, Basel

$0253-4886 / 08 / 0251-0001 \$ 24.50 / 0$

Accessible online at:

www.karger.com/dsu tomy. The $\chi^{2}$ test analysis showed the presence of heterogeneity among studies (Tiffenau index $\chi^{2}=99.97, p=0.03$ ). The sensitivity analysis confirms the validity of our results. Conclusions: Postoperative pulmonary function is better preserved after laparoscopic cholecystectomy than open cholecystectomy.

Copyright $\odot 2008$ S. Karger AG, Basel

\section{Introduction}

Cholecystectomy, surgical removal of the gallbladder, is one of the most common elective procedures performed by general surgeons [1].

Advanced laparoscopic technologies make it possible to remove the gallbladder through a tiny incision at the navel.

The speed with which laparoscopic cholecystectomy has been developed and introduced into routine practice is unprecedented in the history of surgical procedures. The introduction of laparoscopic cholecystectomy induced a significant growth in cholecystectomy rates $[2,3]$.

Laparoscopic cholecystectomy has quickly become a popular alternative to open cholecystectomy.

Prof. Gianfranco Damiani

Department of Public Health, Università Cattolica S. Cuore

Largo F. Vito 1

IT-00168 Rome (Italy)

Tel. +39 06301 5837, Fax +39 06305 3168, E-Mail gdamiani@rm.unicatt.it 
Smaller incisions and the use of laparoscopic techniques allow substantial reductions in pulmonary function to be prevented postoperatively $[4,5]$.

Altogether, laparoscopy is considered a cost-effective technique for the therapy of symptomatic cholecystolithiasis $[6,7]$ showing a number of advantages over laparotomy.

In particular, regarding the dimension of the effectiveness, it is possible to distinguish two different kinds of outcome related to admission period: intra-admission and post-admission outcomes.

According to scientific literature, post-admission outcomes of laparoscopic cholecystectomy are faster return to normal activities and a better quality of life [8-13].

Instead, considering intra-admission outcomes, we found that laparoscopic cholecystectomy is associated with a faster recovery, shorter hospitalization, lower mortality rate, less postoperative pain and a better pulmonary function [13-26]. All these outcomes linked to the postadmission period, reflect the quality of care during the admission. Particularly, the Tiffenau Index, the ratio between forced expiratory volume in $1 \mathrm{~s}\left(\mathrm{FEV}_{1}\right)$ and forced vital capacity (FVC), help to differentiate airflow limitations from restrictive abnormalities [15, 27].

In a more recent view, the predictive value of $\mathrm{FEV}_{1}$, FVC and their ratio about pulmonary complications was underlined. Fuso's and Chetta's reviews support the importance of clinical relevance in assessing these respiratory outcomes. In particular, they reported that patients with lower values of respiratory parameters had an increased risk of pulmonary complications with respect to patients with normal patterns and the reduction of $\mathrm{FEV}_{1}$ was an independent predictor of pulmonary impairment after abdominal surgery [28-30].

Within a project financed by Italian Ministry of Health 'Outcome evaluation in conventional surgery and minimally invasive surgery', we examined the scientific literature on cholecystectomy. The selected articles did not show univocal results about the efficacy of laparoscopic surgery on preserving a better pulmonary function with respect to open surgery. For this reason, we decided to synthesize the results from the scientific literature of postoperative pulmonary function. Therefore, a quantitative synthesis allows making clear which technique better preserves pulmonary function. A meta-analysis of the literature was carried out, concerning the postoperative pulmonary function in both surgical techniques, focused on the Tiffenau Index.

\section{Materials and Methods}

\section{Search Strategy}

An extensive search of the scientific literature was carried out querying electronic databases to identify relevant studies: Cochrane Controlled Trials Register, Database of Abstracts of Reviews of Effects (DARE), Embase, and Pubmed. Literature search covered the period from 1990 to 2007 because the first laparoscopic cholecystectomies were performed in the early 1990s.

The key words used to search articles were associated to interventions, population and outcomes. The following search terms were used:

- Terms related to intervention: 'laparoscopic cholecystectomy', 'open cholecystectomy', 'minimally invasive cholecystectomy'; 'conventional cholecystectomy', 'traditional cholecystectomy', 'minimal access cholecystectomy', 'open versus laparoscopic cholecystectomy'.

- Terms linked to population: 'adult patients', 'surgical patients'.

- Terms related to measured outcomes: 'Tiffenau Index', 'postoperative forced vital capacity and forced expiratory volume in one second', 'postoperative pulmonary function', 'lung function', 'respiratory function', 'respiratory function test'.

A further analysis of the grey literature (those papers that were not published in primary sources) was conducted in generic search engines consulting 'Google scholar' (http://scholar.google. com/) and 'Vivisimo' (www.vivisimo.com), looking for unpublished studies about laparoscopic cholecystectomy.

We integrated electronic searches by hand searching, manually checking the reference lists of relevant articles and contacting experts working in the field to identify any further studies [31].

Studies in English, Italian, French and German were taken into account.

\section{Inclusion Criteria}

Referring to study design, we included exclusively analytical or experimental studies; descriptive studies were excluded.

Regarding exposure criteria, the research focused on comparison between open versus laparoscopic cholecystectomy; we excluded all articles which analyzed different surgical interventions or procedures. Studies which treated acute cholecystectomy and mini-laparoscopic cholecystectomy were also excluded.

Referring to outcome measure, we included in the review studies evaluating postoperative pulmonary function in terms of FVC and $\mathrm{FEV}_{1}$ or the Tiffenau Index.

Studies that focused on different outcomes or those whose $\mathrm{FEV}_{1}$ and FVC values were evaluated in percentages were excluded.

Only adult patients (age $\geq 18$ years) were considered in the selection of the studies. We excluded studies enrolling young patients or children (age $<18$ years).

\section{Quality Assessment}

The methodology of each study was assessed independently by 2 authors (L.P. and A.S.) according to a score based on a 10-point scale, consisting of 5 potential sources of bias [32, 33].

Disagreements were resolved with a third epidemiologist (G.D.) or by consensus. 
Table 1. Characteristics of studies included in the meta-analysis

\begin{tabular}{|c|c|c|c|c|c|c|c|}
\hline Authors [ref.] & Country & Year & Study design & Open & $\begin{array}{l}\text { Laparo- } \\
\text { scopic }\end{array}$ & Spirometry & $\begin{array}{l}\text { Quality } \\
\text { scores }\end{array}$ \\
\hline Bablekos [14] & Greece & 2003 & cohort study & 10 & 18 & $\begin{array}{l}\text { Med-graphics cardiorespiratory } \\
\text { diagnostic systems }\end{array}$ & $8 / 10$ \\
\hline Hasukić [15] & $\begin{array}{l}\text { Bosnia and } \\
\text { Herzegovina }\end{array}$ & 2002 & randomized trial & 28 & 30 & Bodypletizmograf, Germany & $9 / 10$ \\
\hline Hendolin [16] & Finland & 2000 & randomized trial & 22 & 25 & Vitalograph England & $10 / 10$ \\
\hline Joris [ 17] & Belgium & 1992 & cohort study & 15 & 15 & Gould pulmonet 3 & $8 / 10$ \\
\hline Karayannakis [18] & England & 1996 & randomized trial & 40 & 42 & Bodyscreen 2 & $10 / 10$ \\
\hline Kimberley [19] & USA & 1996 & cohort study & 3 & 29 & Vitalo-graph Compact & $7 / 10$ \\
\hline Mimica $[20]$ & Croatia & 2000 & cohort study & 50 & 50 & VICATEST-4 vitalograph & $10 / 10$ \\
\hline Peters $[21]$ & USA & 1993 & cohort study & 9 & 30 & Cybermedic 2 plesthymograph & $7 / 10$ \\
\hline Putensen-Himmer [22] & Austria & 1992 & randomized trial & 10 & 10 & PFT-Horizon & $7 / 10$ \\
\hline Ravimohan [23] & India & 2005 & cohort study & 15 & 40 & Morgan Spiro flow 2000 & $6 / 10$ \\
\hline Rovina [24] & Greece & 1996 & cohort study & 25 & 26 & Flowmate-Jaeger & $8 / 10$ \\
\hline Volpino [25] & Italy & 1998 & randomized trial & 60 & 58 & Vitalograph England & $10 / 10$ \\
\hline Williams [26] & USA & 1993 & cohort study & 10 & 10 & $\begin{array}{l}\text { Med-graphics pulmonary } \\
\text { diagnostic systems spirometer }\end{array}$ & $6 / 10$ \\
\hline
\end{tabular}

We considered the method of allocation to study groups (random, 2, vs. quasi-random, 1 , vs. selected concurrent controls, 0 ), data analysis and presentation of results (appropriate statistical analysis and clear presentation of results, 2 , inappropriate statistical analysis or unclear presentation of results, 1, inappropriate statistical analysis and unclear presentation of results, 0), the presence of baseline differences between the groups that were potentially linked to study outcomes (of particular importance for observational studies; no baseline differences present or appropriate statistical adjustments made for differences, 2 , vs. baseline differences present and no statistical adjustments made, 1, vs. baseline characteristics not reported, 0 ), the objectivity of the outcome (objective outcomes or subjective outcomes with blinded assessment, 2, vs. subjective outcomes with no blinding but clearly defined assessment criteria, 1, vs. subjective outcomes with no blinding and poorly defined, 0 ), and the completeness of followup for the appropriate unit of analysis (90\%, 2, vs. $80-90 \%$, 1, vs. $80 \%$ or not described, 0 ).

The cut-off value for including an article in our meta-analysis was $6 / 10$.

\section{Data Extraction and Data Analysis}

Data were extracted using a standardized form referring to: authors, year of publication, study design, population, interventions, and outcomes related to postoperative pulmonary function (Tiffenau index, $\mathrm{FEV}_{1}, \mathrm{FVC}$ ).

Two authors (L.P. and A.S.) independently extracted data from the papers retrieved and corroborated their findings.

The kappa test was performed to evaluate agreement between the reviewers regarding the quality of the selected studies.

The statistical software Review Manager 4.2 was utilized for performing the meta-analysis [34].

The mean differences were calculated for continuous variables (FVC and $\mathrm{FEV}_{1}$ ) with the $95 \% \mathrm{CI}$.

Postoperative Pulmonary Function after Open vs. Laparoscopic Cholecystectomy
Since the selected studies evaluated FVC and $\mathrm{FEV}_{1}$ using different spirometer devices (table 1), we calculated the standardized mean difference (SMD). According to the definition of the 'Cochrane Handbook for Systematic Reviews of Interventions', SMD is a summary statistic used in meta-analysis when all trials assess the same outcomes, but measure it in a variety of ways. It is necessary to standardize the results of the trials to a uniform scale, before they can be combined. It is calculated as the ratio between difference in mean outcome among groups and the standard deviation of outcomes among participants [35].

Heterogeneity among studies was tested by using a $\chi^{2}$ test at the 0.05 significance level. A random-effects meta-analysis was applied.

A sensitivity analysis was carried out to assess the robustness of our study: we estimated the SMD for the Tiffenau index by excluding the most influential studies on the pooled estimate, i.e. the study with the higher weight and the study with the larger size effect.

\section{Results}

The combined searches resulted in a total of 370 potentially relevant studies identified by abstract and title. Of these identified trials, 357 were excluded because they did not fit our inclusion criteria. In particular, most of them considered different outcomes, different interventions, or Tiffenau Index was not evaluated. Others were excluded because they were descriptive studies or they calculated percentage values of $\mathrm{FEV}_{1}$ and FVC, without providing baseline values (so it was not possible to calculate the means) or ratio between $\mathrm{FEV}_{1}$ and FVC. The ma- 


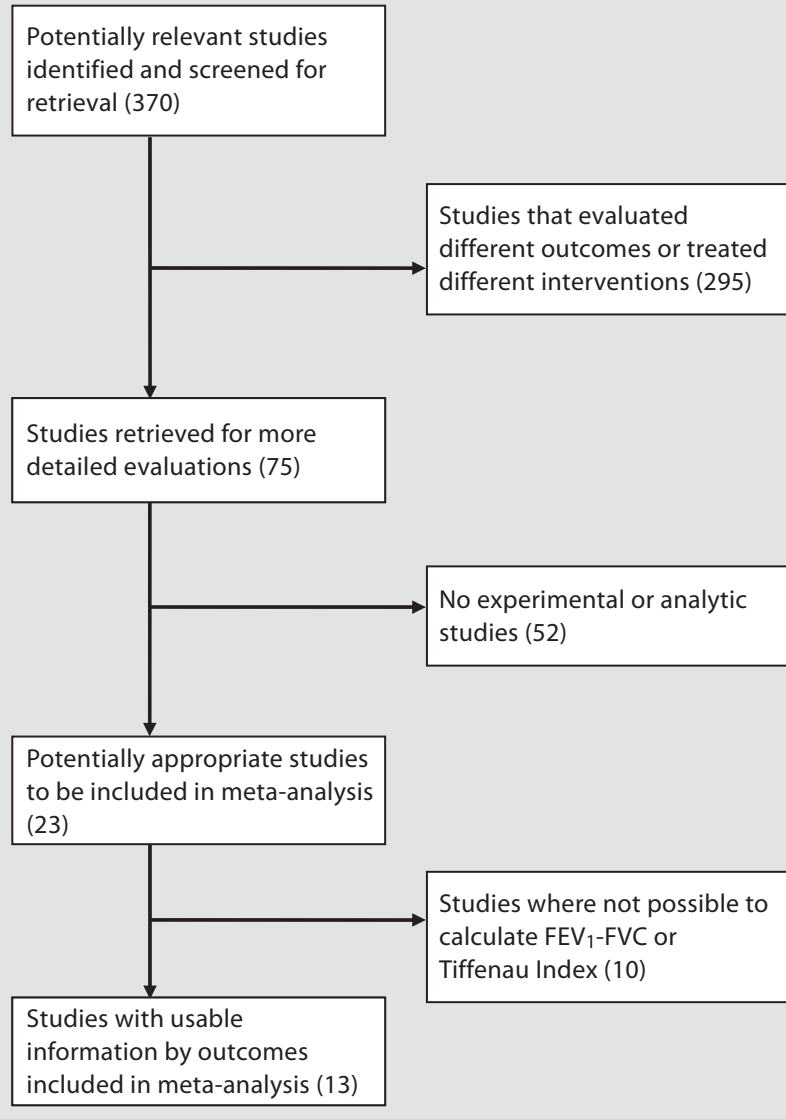

Fig. 1. Flow of studies through the stages of the review strategy.

jority presented unsatisfactory information, considering only laparoscopic values or only vital capacity values. The remaining 13 articles [14-26], whose publication period ranged from 1992 to 2005, met the selection criteria and were included in the meta-analysis (table 1). Among the selected articles, 5 were randomized trials $[15,16,18,22$, $25]$ and the other 8 were nonrandomized [14, 17, 19-21, $23,24,26]$. Thus, the final analysis considered a total of 680 patients: 297 underwent open cholecystectomy and 383 laparoscopic cholecystectomy.

The final agreement on selection of relevant articles was $100 \%$.

Figure 1 shows a summary profile of the search.

The kappa test for quality analysis reports a substantial agreement between the two authors: $\kappa=0.70,95 \%$ CI 0.48; 0.91 for method of allocation to study groups, $\kappa=$ $0.75,95 \%$ CI $0.55 ; 0.95$ for data analysis and presentation of results, $\kappa=0.85,95 \%$ CI $0.69 ; 1.00$ for presence of baseline differences between the groups, $\kappa=0.65,95 \%$ CI $0.42 ; 0.88$ for objectivity of the outcome and $\kappa=0.70,95 \%$ CI 0.48; 0.91 for completeness of follow-up. No articles scored a quality value lower than cut-off (table 1).

The meta-analysis of 13 articles resulted in a SMD of $53 \%$ (95\% CI 0.04, 1.02) for the Tiffenau Index in favor of laparoscopic cholecystectomy (fig. 2).

The $\chi^{2}$ test analysis showed the presence of heterogeneity among studies (Tiffenau index $\chi^{2}=99.97 \mathrm{p}=0.03$ ), so a random effect analysis was performed.

The sensitivity analysis confirms the validity of our results. We repeated the analysis using a fixed-effects model and it showed no differences from the results of a random-effects model.

We then removed the most influential study, showing that the results of the meta-analysis were not conditioned by it (table 2).

Moreover, publication bias was not found by the funnel plot used to identify possible missing studies (fig. 3).

\section{Discussion}

The spread of laparoscopic cholecystectomy has changed the general surgeon's and indeed the public's view of the morbidity associated with abdominal surgery.

Respiratory insufficiency associated with the development of pulmonary function occupies first place on the scale of postoperative morbidity and mortality [25].

The evaluation of pulmonary function reflects the quality of care and the risks of infectious complications during the intra-admission period.

Considering that laparoscopy has a number of implications for patients, health care providers (health care professionals and facilities), the organization of health care, quality of care and society as a whole, it is important to provide quantitative evaluations on this topic for decision-makers.

Because of its potentially strategic influence as a tool for data synthesis in such highly active fields as technology assessment and outcomes research, meta-analysis may affect health care policy in a profound manner [36].

To our knowledge there has been no other meta-analysis comparing pulmonary function after laparoscopic and open cholecystectomy, and even if in the literature there are small studies concerning this topic, our attempt was to provide a synthetic result. Single studies included 


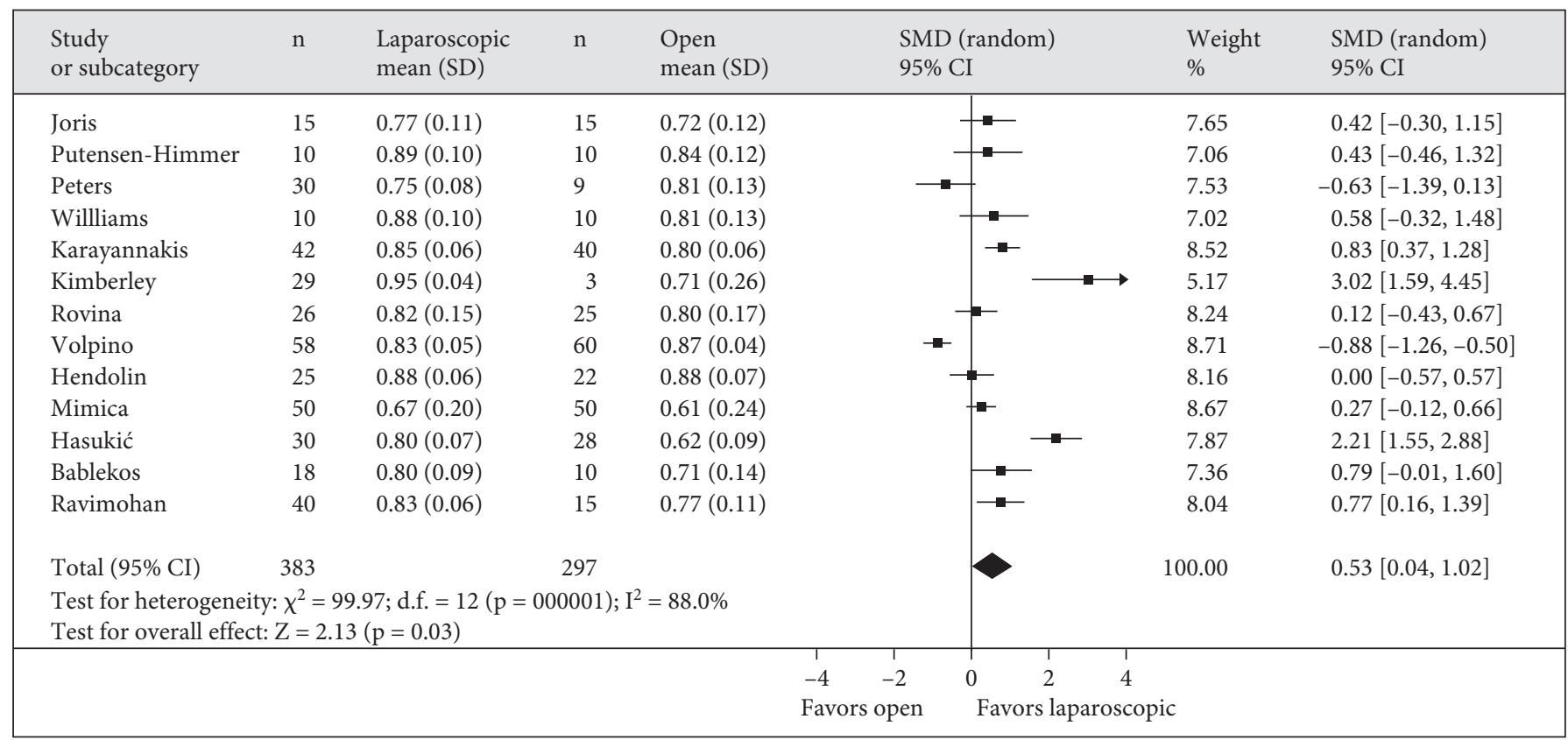

Fig. 2. Forest plot of the Tiffenau Index for laparoscopic vs. open cholecystectomy.

Table 2. Sensitivity analysis

\begin{tabular}{llll}
\hline Random effect model & Fixed effect model & $\begin{array}{l}\text { Analysis without most influential study for } \\
\text { effect size (Kimberly 3.02 CI 95\% }=1.59,4.45)\end{array}$ & $\begin{array}{l}\text { Analysis without most influential } \\
\text { study for weight (Mimica 8.67\%) }\end{array}$ \\
\hline 0.53 & 0.29 & 0.25 & 0.29 \\
CI 95\% $=0.04,1.02$ & CI 95\% $=0.12,0.45$ & CI 95\% $=0.09,0.41$ & CI 95\% $=0.11,0.47$ \\
\hline
\end{tabular}

in our meta-analysis show contrasting or nonsignificant results in terms of FVC and $\mathrm{FEV}_{1}$ values or the Tiffenau Index and they also assess these surrogates on different scales, but our work provides a unique quantitative result in favor of laparoscopic cholecystectomy.

Our meta-analysis suggests that the laparoscopic compared with the laparotomic technique can reduce airflow limitations and avoid postoperative pulmonary restriction.

This should translate into a lower risk of respiratory complications, particularly in patients with underlying pulmonary disease [23].

This evidence underlines the effectiveness of laparoscopic cholecystectomy since it increases the knowledge on this specific topic and supplies a quantitative synthetic result, through a strict and reproducible technique. The studies under investigation were mostly nonran-

Postoperative Pulmonary Function after Open vs. Laparoscopic Cholecystectomy

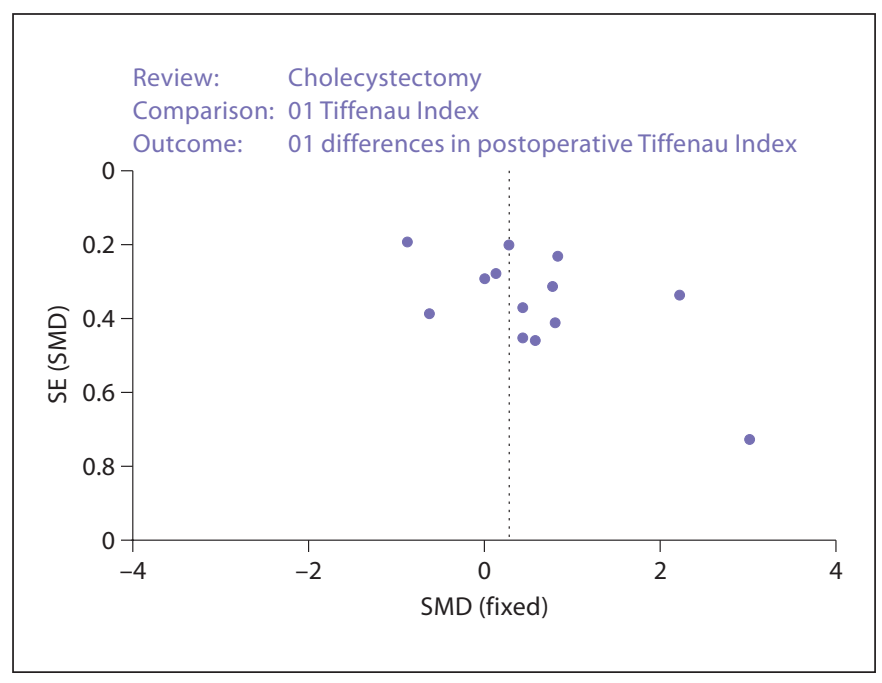

Fig. 3. Funnel plot. 
domized (only 5 were randomized trials), therefore the results have to be interpreted with caution.

Another relevant result of our meta-analysis is the increase of the precision of the estimated effect measure reported in studies with a small sample size.

Although studies of different settings were included in our review, we used the quality score analysis to minimize the distortions related to this phenomenon.

Taking all these implications into account, we can affirm that laparoscopic cholecystectomy can preserve the postoperative pulmonary function better than open surgery, so our meta-analysis promotes the adoption of this technique as a safer alternative to traditional surgery.

Laparoscopic cholecystectomy is also associated with faster recovery, shorter hospitalization, lower mortality rate, less postoperative pain, faster return to normal activities and a better quality of life than open cholecystectomy [8-12].
Our results on the effectiveness of postoperative pulmonary function after laparoscopic cholecystectomy should be included in a health technology assessment evaluation in order to go beyond the singular experiences presented in the literature, such as cost-effectiveness evaluations.

The method of meta-analysis should be implemented in a context of health technology assessment evaluations for other minimally invasive procedures, as well as laparoscopic cholecystectomy, allowing synthetic results to be obtained and increasing the precision of the estimated effect measure.

We think that large surgical trials (RCTs) and further systematic reviews of individual patients' data for adequate pooling of results and for addressing heterogeneity should be carried out in order to provide better and updated evidence on this topic.

\section{References}

1 McMahon A, Fishbacher C, Frame S, MacLeod M: Impact of laparoscopic cholecystectomy: a population-based study. Lancet 2000;356:1632-1637.

$\checkmark 2$ Arnold C, Daley M, Pappas J, Henderson WG, Shukri K: Growing use of laparoscopic cholecystectomy in the national veterans affairs surgical risk study: effects on volume, patients selection, and selected outcomes. Ann Surg 1998;227:12-24.

$\checkmark 3$ Urbach D, Stukel T: Rate of elective cholecystectomy and the incidence of severe gallstones disease. JAMC 2005; 172:1015-1019.

$\checkmark 4$ Ferguson MK: Preoperative assessment of pulmonary risk. Chest 1999;115:58-63.

$\checkmark 5$ Rademaker BM, Ringers J, Odoom J, de Wit L, Kalkman CJ, et al: Pulmonary function and stress response after laparoscopic cholecystectomy: comparison with subcostal incision and influence of thoracic epidural analgesia. Anesth Analg 1992;75:381-385.

-6 McKellar DP, Johnson RM, Dutro JA, Mellinger J, Bernie WA, Peoples JB: Cost-effectiveness of laparoscopic cholecystectomy. Surg Endosc 1995;9:158-163.

7 Bass E, Pitt H, Lillemoe KD: Cost-effectiveness of laparoscopic cholecystectomy versus open cholecystectomy. Am J Surg 1993;165: 466-471.

$>8$ Sawyers L: Current status of conventional (open) cholecystectomy versus laparoscopic cholecystectomy. Ann Surg 1996;223:1-3.

$\checkmark 9$ Velanovich V: Laparoscopic versus open surgery. A preliminary comparison of quality of life outcomes. Surg Endosc 2000;14:16-21.
10 Quintana Q, Cabriada J, Arostegui I, Lopez de Tejada I, Bilbao A: Quality-of-life outcomes with laparoscopic vs. open cholecystectomy. Surg Endosc 2003;17:1129-1134.

11 Chen L, Tao SF, XU Y, Fang F, Peng S: Patients' quality of life after laparoscopic or open cholecystectomy. J Zhejiang Univ Sci 2005;6B:678-681.

12 Topcu O, Karakayali F, Kuzu MA, et al: Comparison of long-term quality of life after laparoscopic versus open cholecystectomy. Surg Endosc 2003;17:291-295.

13 Keus F, de Jong JA, Gooszen HG, van Laarhoven CJ: Laparoscopic versus open cholecystectomy for patients with symptomatic cholecystolithiasis. Cochrane Database Syst Rev 2006.

14 Bablekos GD, Roussou T, Rasmussen T, Vassiliou MP, Behrakis PK: Postoperative changes on pulmonary function after laparoscopic and open cholecystectomy. Hepatogastroenterology 2003;50:1193-1200.

15 Hasukić S, Mesić D, Dizdarević E, Keser D, Hadziselimović S, Bazardzanović M: Pulmonary function after laparoscopic and open cholecystectomy. Surg Endosc 2002;16: 163-165.

16 Hendolin HI, Paakkonen ME, Alhava EM, Tarvainen R, Kemppinen T, Lahtinen P: Laparoscopic or open cholecystectomy: a prospective randomised trial to compare postoperative pain, pulmonary function, and stress. Eur J Surg 2000;166:394-399.

17 Joris J, Cigarini I, Legrand M, et al: Metabolic and respiratory changes after cholecystectomy performed via laparotomy or laparoscopy. Br J Anaesth 1992;69:341-345.
18 Karayannakis AJ, Makri G, Mantzioka A, Karousos D, Karatzas G: Postoperative pulmonary function after laparoscopic versus open cholecystectomy. Br J Anaesth 1996;77: 448-452.

19 Kimberley N, Kirrkpartick S, Watters J: Alterations in respiratory mechanic after laparoscopic and open cholecystectectomy. Can J Surg 1996;39:312-316.

20 Mimica Z, Biocic M, Bacic A, et al: Laparoscopic and laparotomic cholecystectomy: a randomized trial comparing postoperative respiratory function. Respiration 2000;67: 153-158.

21 Peters JH, Ortega A, Lehnerd S, et al: The physiology of laparoscopic surgery: pulmonary function after laparoscopic cholecystectomy. Surg Laparosc Endosc 1993;3:370374.

22 Putensen-Himmer G, Putensen C, Lammer H, Lingnau W, Aigner F, Benzer H: Comparison of postoperative respiratory function after laparoscopy or open laparotomy for cholecystectomy. Anaesthesiology 1992;72: 675-680.

23 Ravimohan M, Lileswar K, Ravul J, Singh R, Jindal R: Postoperative pulmonary function in laparoscopic versus open cholecystectomy: a prospective, comparative study. Indian J Gastroenterol 2005;24:6-8.

24 Rovina N, Bouros D, Tzanakis N, Velegakis M, Kandilakis S, et al: Effects of laparoscopic cholecystectomy on global respiratory muscle strength. Am J Resp Crit Care Med 1996;153:458-461. 
25 Volpino P, Cangemi V, D’Andrea N, Cangemi B, Piat G: Hemodynamic and pulmonary changes during and after laparoscopic cholecystectomy. Surg Endosc 1998;12:119-123.

-26 Williams MD, Sulentich SM, Murr PC: Laparoscopic cholecystectomy produces less postoperative restriction of pulmonary function than open cholecystectomy. Surg Endosc 1993;7:489-492.

-27 Schauer P, Luna J, Ghiatas A, Glen M, Warren J, et al: Pulmonary function after laparoscopic cholecystectomy. Surgery 1993;114: 389-399.

-28 Fuso L, Cisternino L, Di Napoli A, Di Cosmo V, Tramaglino LM, et al: Role of spirometric and arterial gas data in predicting pulmonary complications after abdominal surgery. Resp Med 2000;94:1171-1176.
29 Chetta A, Panagiota T, Marangio E, Carbognani $P$, Bobbio A, et al: Respiratory effects of surgery and pulmonary function testing in the preoperative evaluation. Acta Biomed 2006;77:69-74.

30 Lawrence VA, Page CP, Harris GD: Preoperative spirometry before abdominal operations: a critical appraisal of its predictive value. Arch Intern Med 1989;149:280-285.

31 McManus RJ, Wilson S, Delaney BC, Fitzmaurice D, Hyde C, et al: Review of the usefulness of contacting other experts when conducting a literature search for systematic review. BMJ 1998;317:1562-1563.

32 Hunt DL, Haynes RB, Hanna SE, Smith K: Effects of computer-based clinical decision support systems on physicians performance and patient outcomes. J Am Med Assoc 1998; 280:1339-1346.
33 Haynes RB, Walker C: Computer-aided quality assurance. Arch Intern Med 1987; 147:1297-1301.

34 Review Manager (RevMan) [Computer program]: Version 4.2 for Windows. Oxford, The Cochrane Collaboration, 2003.

35 Higgins JPT, Green S (ed): Cochrane Handbook for Systematic Reviews of Interventions 4.2.6 [updated September 2006]; in: The Cochrane Library, Issue 4, 2006. Chichester, Wiley, 2006.

36 Vatz JB: Meta-analysis: apples and oranges, or fruitless - statistical method for systematic synthesis of health care management and policy research findings - column. Physician Exec 1991;17:40-42. 